\title{
Multi-scale and multi-frequency studies of cosmic ray air shower radio signals at the CODALEMA site
}

\author{
R. Dallier ${ }^{a, b *}$, J.L. Béney ${ }^{a}$, H. Carduner ${ }^{a}$, D. Charrier ${ }^{a}$, L. Denis ${ }^{b}$, F. Gaté ${ }^{a}$, \\ A. Lecacheux ${ }^{c}$, V. Marin ${ }^{a}$, L. Martin ${ }^{a, b}$, B. Revenu ${ }^{a}$, D. Torres Machado ${ }^{d}$ \\ ${ }^{a}$ SUBATECH - Ecole des Mines de Nantes - CNRS/IN2P3 - Université de Nantes, 4 rue Alfred \\ Kastler, 44307 Nantes cedex 3, France \\ ${ }^{b}$ U.S. Nançay - Observatoire de Paris, CNRS/INSU, 18330 Nançay, France \\ ${ }^{c}$ LESIA - Observatoire de Paris, CNRS/INSU, 5 place Jules Janssen, 92190 Meudon, France \\ ${ }^{d}$ Universidade Federal do Rio de Janeiro, Av. Pedro Calmon, 550 - Cidade Universitária, Rio de \\ Janeiro - RJ, 21941-901, Brazil \\ E-mail: richard. dallierdsubatech.in2p3. fr
}

Since 2003, the Nançay Radio Observatory hosts the CODALEMA experiment, dedicated to the radio detection of cosmic ray induced extensive air showers. After several instrumental upgrades, CODALEMA is now composed of:

- 57 self-triggering radio detection stations working in the [20-200] $\mathrm{MHz}$ band, over $1 \mathrm{~km}^{2}$;

- an array of 13 scintillators acting as a particle detector;

- a compact array of 10 cabled antennas, triggered by the particle detector, to test the capabilities of a phased antenna cluster to cleverly select air shower events.

In addition, CODALEMA supports the EXTASIS project, aiming at detecting the expected lowfrequency signal produced by the sudden deceleration of the air shower particles hitting the ground. Beside these dedicated arrays, the Nançay site will host the NenuFAR radio telescope (recognized as a SKA pathfinder), made of 1824 dual crossed-polarization antennas similar to the CODALEMA ones. All these arrays present different antenna density and extent, and could be operated in a joint mode to record simultaneously the radio signal coming from an air shower. Therefore, the upgraded CODALEMA facilities could offer a complete description of the air shower induced electric field at small, medium and large scale, and over an unique and very wide frequency band (from $\sim 2$ to $\sim 200 \mathrm{MHz}$ ). The use of multi-band detectors combined with composite trigger algorithms could help boosting the radio detection technique as a candidate for an accurate composition measurement with a high efficiency, or in the frame of a further very large cosmic ray observatory. We describe the current and foreseen instrumental set-up together with the prospective developments of the radio detection technique.

The 34th International Cosmic Ray Conference,

30 July- 6 August, 2015

The Hague, The Netherlands

\footnotetext{
${ }^{*}$ Speaker.
} 


\section{Radio detection: performances and limitations}

Radio detection of cosmic ray extensive air showers (EAS) is based on the measurement of the electric field generated at high altitude by the charged particles in the shower. The shower history, corresponding to its evolution in time, is contained in the radio signal and can be retrieved with a high effective duty cycle. Nowadays, thanks to powerful simulation codes [1] we have a very good understanding of the emission mechanisms, from the main geomagnetic effect [2] down to the timevarying charge-excess radiation [3]. It has also been demonstrated that all important properties of extensive air showers can in principle be deduced from radio measurements, in particular the arrival direction, the energy, and recently several mass-sensitive observables $[4,5]$. The radio technique is now used in a flurry of experiments throughout the world, among which AERA [6], LOFAR [7] and CODALEMA are the most productive. These various instruments present different bandwidth and detector densities: AERA measures showers at frequencies between $30 \mathrm{MHz}$ and $80 \mathrm{MHz}$, while CODALEMA/EXTASIS bandwidth extends from $2 \mathrm{MHz}$ up to $200 \mathrm{MHz}$; LOFAR has both a low frequency $(30-80 \mathrm{MHz})$ and high frequency window $(110-250 \mathrm{MHz})$, but they cannot be used simultaneously; on AERA, the detection technique is tested over a very large area $\left(\sim 17 \mathrm{~km}^{2}\right)$, with a sparse array of autonomous radio detector stations; contrariwise, LOFAR features a very dense core of radio antennas, externally triggered by a particle detector, over an area of $\sim 0.2 \mathrm{~km}^{2}$ allowing one to study the electric field profile of individual air showers with very high precision.

However, either triggered by ground particle arrays (LOFAR) or with standalone radio detectors achieving EAS detection triggered by the radio signal itself (CODALEMA and AERA), these experiments still face strong limitations: 1) Difficulties for standalone instruments arise from parasitic radio transients. On the contrary, externally triggered systems are almost blind to highly inclined EAS, due to the particle detectors, while the radio signal is expected to be higher for inclined showers. 2) In the state of art, the detection range of radio antennas in the [30-80] $\mathrm{MHz}$ band is still too short (a few hundreds of meters) to envision a very large array - typically thousands of $\mathrm{km}^{2}$ - made of this type of radio detection system at a reasonable cost. 3) Current methods for the determination of the "radio $X_{\max }$ " are based on the reconstruction of the radio LDF. The larger the antenna density within the electric field footprint at ground, the best the accuracy on $X_{\max }$, notably due to a good spatial sampling of the expected radio Cerenkov ring of the footprint (case of LOFAR). But with a sparse array, rapid variations of the electric field profile may be smoothed or even not measured (case of AERA), degrading the accuracy on the reconstructed $X_{\max }$. 4) Current operated antennas are horizontally polarized along the East-West (EW) and North-South (NS) directions, and under the "far field assumption" the vertical, third component, can be reconstructed from the other two. This assumption has never been verified experimentally, though measuring the complete electric field could help refining the conversion of the electric field value to an energy.

Overcoming those limitations would require new instrumental developments. Therefore at the CODALEMA site at the Nançay radio observatory, we have chosen to explore some possible solutions to each of the above problems: 1) To improve autonomous detection, clever triggering algorithms may be used, thanks to the online combination of several antennas in a clustered way (composite trigger). This could also allow one to get rid of a particle detector. 2) Using such a phased combination of several antennas will naturally increase the detection range. The latter is moreover larger at low frequencies, where the air shower radio signal maximum is expected (around 
$10 \mathrm{MHz}$ ). Furthermore, the air shower "sudden death" signal, when the last secondary particles of the shower disappear at ground, is also foreseen below $10 \mathrm{MHz}$ [9], giving a completely new and different observable of the charge contents of the shower. 3) On the opposite, above $120 \mathrm{MHz}$, a strong dependence of the signal strength with the distance to the shower axis has been noticed [10], and the radio Cerenkov ring can be well observed $[11,5]$, which could help solving the problem of the antenna density at short distance of the shower axis. The electric field profile sampled up to the higher frequencies where the rapid variations are seen could then be better defined. 4) Several attempts have been made to measure the vertical polarization of the electric field. However, none has given convincing results. A revision of the concept of the three-dimensional measurement of the electric field should be done.

In this paper, we will shortly describe the Nançay experimental site facilities devoted to radio detection of UHECR working at various frequencies, various space scales and electric field polarizations, accompanied by their perspectives.

\section{The Nançay facilities and the CODALEMA/EXTASIS experiments}

\subsection{CODALEMA}

Since the first attempts in 2002, the CODALEMA experimental setup in Nançay has evolved a lot and is nowadays an experiment composed of 3 arrays of detectors: the particle detector array, 57 standalone radio detectors and a cabled array of antennas (so-called "compact array") aiming at building a composite, purely radio trigger. Fig. 1, left, shows the map of the current setups.

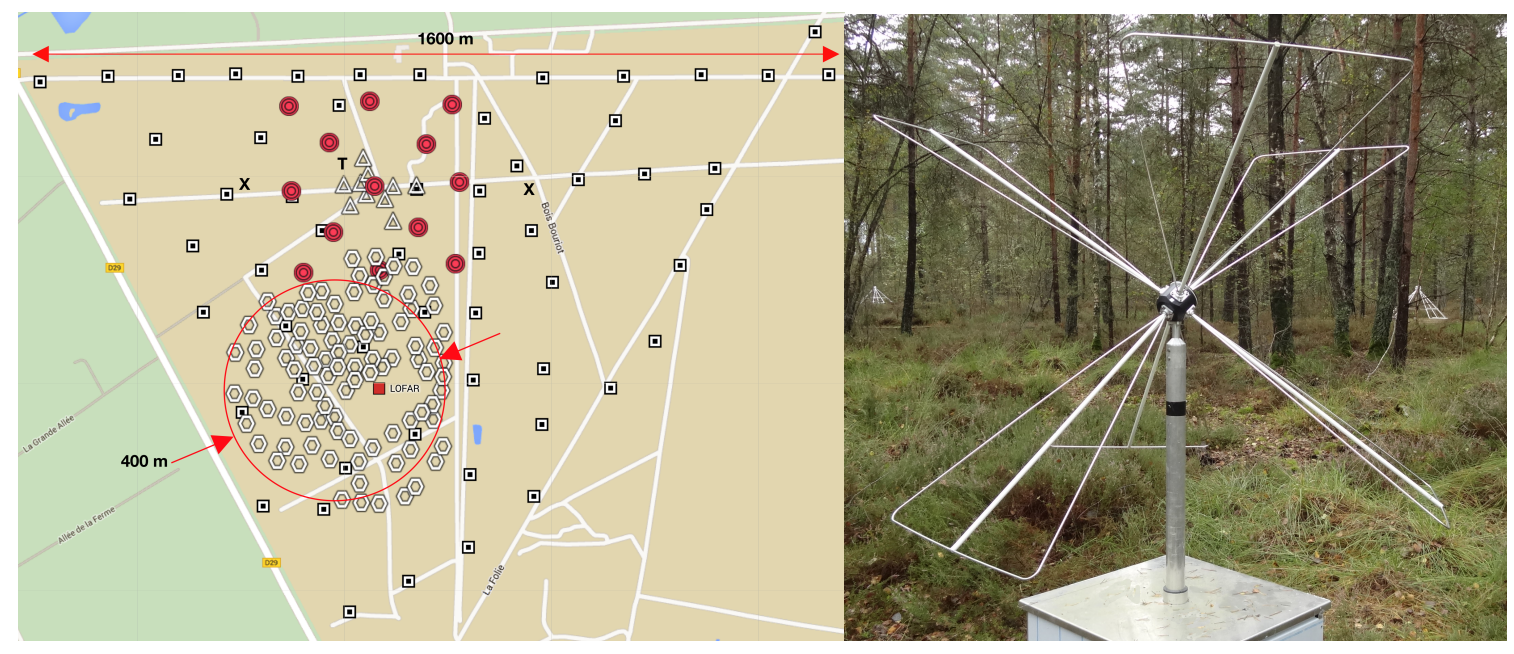

Figure 1: Left: map of the Nançay observatory (North on top). Squares feature the 57 CODALEMA autonomous radio detection stations, red disks the 13 scintillators, triangles the 10 antennas of the compact array, hexagons the 96 mini-arrays of NenuFAR around the LOFAR station. X are the low-frequency antennas and $\mathrm{T}$ is the tripole. Right: the tripole antenna.

\subsubsection{The Scintillator Detector Array (SD)}

The goal of the particle detector is to provide the arrival direction, the size, the core location of the atmospheric shower and an estimation of the energy of the incident cosmic ray. It also provides 
a master trigger which can be possibly broadcasted to any other system throughout the observatory, among others the compact array of antennas and a dedicated datation station holding the same GPS module as the radio stations described later. The particle detector array is composed of 13 scintillators located on a grid with a variable spacing (approximately $120 \mathrm{~m}$ on the diagonal and $170 \mathrm{~m}$ on the NS and EW directions). It covers a $340 \times 340 \mathrm{~m}^{2}$ area whose center roughly corresponds to the former cabled radio array center. The particle detector geometry has been designed so that its energy threshold is located at the knee energy, and the array reaches its maximum efficiency for an energy of $10^{16} \mathrm{eV}$, with a sensitivity level of 1 particle per $\mathrm{m}^{2}$.

\subsubsection{The standalone antenna array}

57 standalone radio detection stations have been installed over $1 \mathrm{~km}^{2}$, with an average spacing of about $150 \mathrm{~m}$ between stations. The latter feature 2 crossed-polarization "Butterfly" antennas also used later on AERA (described in [12]), in the NS and EW directions. Each of both input channels is split in two equal power parts. One part is amplified and filtered by an analog, passive filter in the [35-80] and [150-200] MHz bands.

The filtered signal is compared to a voltage threshold, remotely or automatically adjustable. If the threshold is passed on one or both of the input channels, the level 1 (T1) triggering condition is fulfilled and the event is dated by a GPS board. The daily T1 number on a single station is about $10^{6}$. The other part of the signal (full band) continuously feeds a digitizer board, whose circular memory is frozen only when a T1 arises. A second triggering level (T2) is applied depending on the digitized signal characteristics [8], analyzed on-line by a local PC, which reduces the initial trigger rate by a factor of 1000, therefore getting rid of the most prominent noise sources. A third triggering level (T3) can be applied at the array scale, selecting only interesting

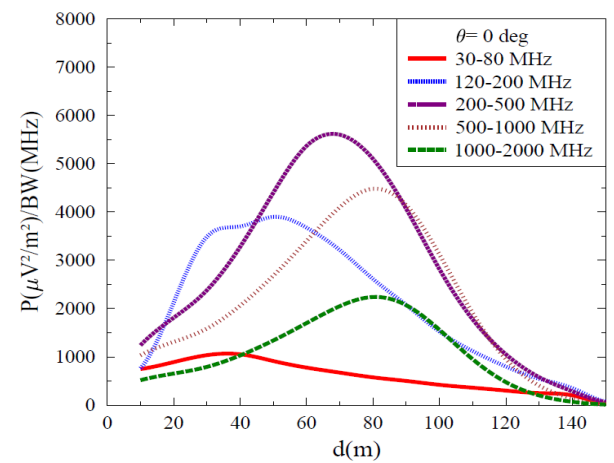

Figure 2: Electric field distribution as a function of the distance to the shower axis, for various frequency bands. The shower is from a vertical proton at $10^{17}$ (from [13]). events having hit several stations simultaneously and constituting the air shower candidates. On average, for an antenna multiplicity of at least 5 , about 2 events a day are finally selected as cosmic ray events in time coincidence with the particle detector ones, above $10^{17} \mathrm{eV}$. If the $\mathrm{FM}$ radio band is not usable due to the large number of emitter lines, a very wide bandwidth is still available, from $20 \mathrm{MHz}$ to $85 \mathrm{MHz}$ and from $110 \mathrm{MHz}$ to $200 \mathrm{MHz}$, thanks to the response of the Butterfly antenna and the fast digitizing system (1 GS/s). CODALEMA is currently the only radio experiment able to access such a wide band at once. The high frequencies could be of paramount interest to sample the Cerenkov ring. As shown on Fig. 2 from [13], close to the axis the expected Cerenkov signal in [120-200] MHz is much higher than in the usual band [30-80] $\mathrm{MHz}$ (with which it competes, giving a "double-peak-like" structure), and furthermore its variation with the distance to the axis is faster. Unless a high antenna density is used (LOFAR case), it is difficult to correctly sample the electric field profile in [30-80] MHz, while using the information above $120 \mathrm{MHz}$ could greatly help determining the later with only few antennas spaced of few tens of meters (CODALEMA case). This is currently studied on real detected events simulated with the SELFAS code [5]. 
Recently, a three-fold antenna system has been installed nearby the center of the particle detector. Its goal is to measure the complete electric field of air showers, i.e along 3 polarizations EW, NS and vertical. Indeed on all currently operating radio detection arrays, only the horizontal polarizations are measured, either directly along NS and EW directions or later projected on these axes. This allows reconstructing the vertical electric field component provided there is no significant longitudinal component (far-field assumption). But though strong hints based on the theories of air shower radio emission tend to validate this hypothesis, the latter has never been verified, probably due to the low performances of the vertically polarized antennas used by now. We chose to reconsider the problem and to use 3 regular Butterfly antennas equipped with the LONAMOS LNA, which have shown on CODALEMA and AERA their very good performances. The main difficulty lying in the mechanical holding of such a triple antenna system along the defined polarization axes, a special mounting has been done, on which two rotations are applied around the NS and vertical axes to form a direct trihedron inclined of $45^{\circ}$ (Fig. 1, right). Triggered by the particle detector, the 3 signals are recorded with the same type of electronics as for the standalone stations. We have now to properly deconvolve these together with the regular autonomous station signals recorded at the same time, to derive conclusions about the initial hypothesis.

\subsubsection{A Compact Array as a composite triggering system}

As pointed out in section 1, two majors problems occur with a sparse array of antennas or standalone stations in the current band 30-80 MHz: the detection range is quite short (few hundreds of meters) and the trigger selectivity is low in standalone mode. To study possible solutions to both these problems, we have installed since mid 2013 a cluster of 10 antennas in dual linear horizontal polarisation, distributed over a square of $150 \mathrm{~m} \times 150 \mathrm{~m}$ ( 24 to $146 \mathrm{~m}$ spacing). This cluster can operate in two observing modes: 1) SD triggered, 20 channels recorded, $6 \mu$ s snapshots with a $400 \mathrm{MHz}$ ADC, on a bandwidth of [10-200] MHz; 2) self detecting mode (development in progress), with a continuous sampling of (up to) 8 channels, in circular polarisation (linear output added in quadrature), with an $\mathrm{ADC}$ at $100 \mathrm{MHz}$, a bandwidth of $50 \mathrm{MHz}$ and a real-time software using a 5 Tflops GPU. The SD triggered mode is used to test, off-line, the reconstruction algorithms intended to be implemented on-line in self-detecting mode. Off-line, about 1000 radio events from 80000 SD triggers have been reconstructed by now, allowing one to finely tune the reconstruction algorithms (see Fig. 3). The beam-forming method helps to avoid triggering on natural and man made transient noise and moreover increases the sensitivity of antennas by a factor of $\sqrt{N}$ ( $N$ : number of antennas in the cluster). We are currently developing the self-triggering, on-line detection scheme based on the continuous sampling of antenna output, stored in a ring buffer. A pre-conditioning is made by Fourier transforming each channel and band pass filtering, then we synthesize a circularly polarized channel by using a wide band quadrature hybrid circuit. The processor computes the signal envelop for each successive time-window corresponding to the array time aperture $(500 \mathrm{~ns})$. It generates $\sim 2000$ beams on the sky $\left(\sim 2^{\circ}\right.$ apart) via beam forming, in which a search for beam(s) containing signal above some intensity threshold is conducted. Additional criteria (e.g. rise time, known RFI direction) may be used to increase the selectivity.

\subsection{NenuFAR}

The project "NenuFAR" - the LOFAR Super Station (LSS) in Nançay [14] - will be installed 

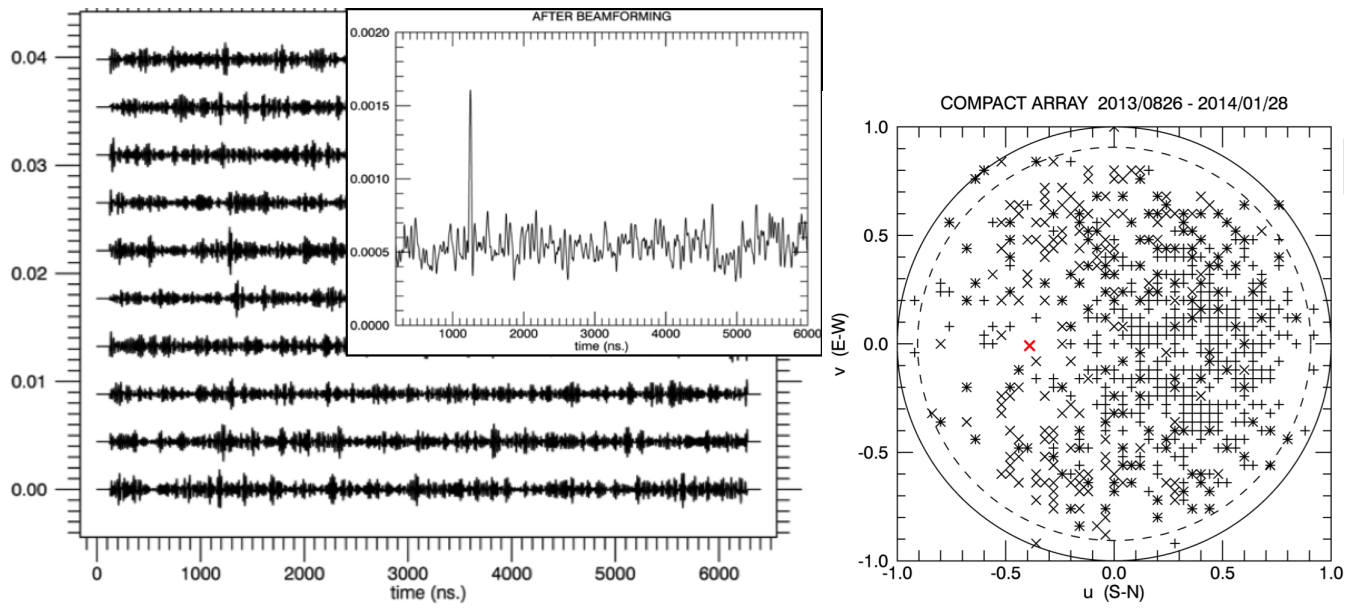

Figure 3: Left: example of EAS event seen by the Compact Array. In the 10 circularly polarized channels (partially hidden), the signal is not visible, but clearly appears after the beamforming procedure, at $1200 \mathrm{~ns}$ (insert). Right: the sky map of reconstructed events, obtained by a blind search off-line conducted over all triggered events. Crosses feature events detected on NS polarization, plus signs on EW polarization. The red cross features the direction of the local magnetic field.

at the Nançay Radio Observatory, which hosts a regular international LOFAR station (FR606). LSS/NenuFAR consists of an extension to the standard LOFAR local station including 96 miniarrays of 19 crossed-dipole antennas (hereafter $\mathrm{MA}_{19}$ ), analogically phased in the $\sim[10-87] \mathrm{MHz}$ frequency window and connected to the 96 dual-polarization receivers of the LOFAR backend. The 3648 dipoles will be positioned within a circle of 400 m diameter ( $~ 5$ LOFAR station diameters). NenuFAR will be used both as part of the LOFAR network in LSS, and as a standalone instrument with large instantaneous sensitivity ( $\sim 2 \times$ LOFAR's core) and wide frequency band. Standalone measurements will be possible due to a dedicated receiver, allowing NenuFAR to be used simultaneously in both modes, within the instantaneous field of view of the beam formed at the $\mathrm{MA}_{19}$ scale (10 to $50^{\circ}$ from 10 to $87 \mathrm{MHz}$ ). As stationary beam-forming is penalizing for cosmic ray detection, a dedicated acquisition channel will be implemented to extract the signal from one single antenna of each of the $96 \mathrm{MA}_{19}$ before beam-forming, and an external trigger input will be available to trigger NenuFAR by CODALEMA during regular astronomical operation. This will offer a high antenna density ( 96 antennas in $0.125 \mathrm{~km}^{2}$ ), though less than the one of LOFAR but large enough to sample the electric field profiles in the same way and compare it to the ones obtained with the surrounding and included standalone stations of CODALEMA.

\subsection{EXTASIS and the low-frequency components}

The coherent emission due to the variation of the transverse current in the shower is expected to be the dominant effect in the global emission of the charged particles of the shower. All the simulation codes show that the maximum of emission peaks at a frequency of $\sim 10 \mathrm{MHz}$, whatever the energy and the geometry of the shower are. Unfortunately, to avoid strong AM and FM lines, all the recent experiments of radio detection of UHECR use a rather "clean" band located in between (i.e $\sim[30-80] \mathrm{MHz}$ ). However, up to the 90's several detections at low and very low frequencies (see [9] and references herein) have shown that the signal has a higher amplitude below $10 \mathrm{MHz}$ than 
above $20 \mathrm{MHz}$, but the various mechanisms proposed to explain this results were not satisfactory. We therefore chose to reinvestigate low frequency measurements, by adding dedicated antennas within CODALEMA and using the SELFAS simulation code to figure out what could be the emission mechanism. Especially, we will focus on the signal predicted by SELFAS at low frequencies $(<5 \mathrm{MHz})$ and appearing when the shower front hits the ground. This is the goal of the EXTASIS project (EXTinction of Air Shower Induced Signal).

The new mechanism proposed in [9] is the coherent emission of the secondary electrons in excess in the shower front when reaching the ground level. These particles suffer a sudden deceleration when they disappear below the ground level; we propose to call this mechanism the sudden death mechanism, which creates the sudden death pulse (SDP). This signal could be of several interests: 1) Let $t=0$ be the time of impact at ground. The SDP will arrive at the antenna at $t_{S D P}=d_{\text {ant }} / c$, where $d_{\text {ant }}$ is the shower core to antenna distance and $c$ the speed of light. With 3 antennas at least, a simple reconstruction of the shower core location can be made. 2) Dating both the regular pulse in [30-80] $\mathrm{MHz}$ and the SDP offers an intrinsic time scale within the shower, the first being related to the maximum of the particle production rate in the shower (strongly correlated to $X_{\max }$ ) and the latter at ground on the axis. 3) It is shown that the SDP amplitude decreases as the distance to the source, thus the shower core, conversely to the regular signal decreasing exponentially. Long range detection is thus favored at low frequencies. 4) Being produced by the last charges in the shower, it gives access to the number of $e^{+} / e^{-}$at ground. Detecting the SDP would give important hints on the emission processes and help disentangling between the various mechanisms proposed so far (among them, the transition radiation of particles at ground level).

In order to detect a low frequency pulse, two antennas have recently been installed within CODALEMA. Their acquisition triggered by the particle detector is made thanks to a digital oscilloscope (18 MS/s, $1 \mathrm{~ms}$ record, 8 bit) in the frequency band [2-5] MHz. The antennas are classical Butterfly antennas with a modified LONAMOS LNA, adapted for the frequency range, on a NorthSouth horizontal polarization and a vertical one, since the SDP is expected to be mainly vertically polarized. They are located on $7 \mathrm{~m}$ high poles, $600 \mathrm{~m}$ apart from each other on an EW line, at equal distance from the particle detector center. After a commissioning phase with these 2 precursor antennas, at least 5 more should be installed to triangulate and recover the shower core location.

\section{Conclusion}

The Nançay radio observatory now offers a variety of instruments, either dedicated or not, for the radio detection of high energy cosmic rays. The main goal of this instrumental setup is to explore new or neglected ways of exploitation of the radio signal emitted by EAS, to fill the remaining gaps of the current detection method. Taking advantage of the very wide bandwidth of the CODALEMA standalone station, we propose an alternative concept able to compensate for the large detector density currently necessary to reach a good sensitivity to the $X_{\max }$. Leant onto the 3 arrays constituting CODALEMA (particle detector array, standalone antenna array and compact antenna array), the EXTASIS project aims at detecting the low frequency counterpart of the regular EAS radio signal, and the so-called sudden death pulse expected to occur at the sudden deceleration of the air shower particles at ground. Beside, thanks to a new design of tripole antenna, a study of the total electric field emitted between 20 and $200 \mathrm{MHz}$ will be led, in order to verify the standard 
far-field hypothesis. At last, the NenuFAR instrument could be used in a slave, externally triggered mode, to sample the ground electric field profile with a high antenna density and correlate the measurements with the ones obtained on a large bandwidth with the standalone stations. Table of Fig. 4 summarizes the various frequency bands and space scales of the instruments constituting the Nançay EAS detection facilities.

\begin{tabular}{|c|c|c|c|c|c|c|c|}
\hline & \multicolumn{7}{|c|}{ Frequency $(\mathrm{MHz})$} \\
\hline \multirow{2}{*}{ 互 27 to 50} & 2 & $\mathrm{AM}$ & 20 & 80 & FM & 120 & 200 \\
\hline & & & 27( & $\mathrm{km}^{2}$ & & & \\
\hline 150 & & & \multicolumn{5}{|c|}{$\begin{array}{c}\text { CODALEMA-3 (+3D antenna) } \\
57+1 / 1 \mathrm{~km}^{2}\end{array}$} \\
\hline 600 & $\begin{array}{c}\text { EXTASIS } \\
2(3) / 0.15 \mathrm{~km}^{2}\end{array}$ & & & & & & \\
\hline
\end{tabular}

Figure 4: Summary table of scales and frequencies of the EAS radio detection facilities at the Nançay Observatory. The scale is an average of the distance between two adjacent detectors (for NenuFAR, the step is variable). The number of antennas (and foreseen antennas) is indicated, as well as the covered area. The AM and FM bands are partially accessible for CODALEMA but intentionally cut in the analysis process.

\section{References}

[1] T. Huege, M. Ludwig and C.W. James, AIP Conference Proceedings 1535128 (2012)

J. Alvarez-Muñiz, W.R. Carvalho and E. Zas, Astropart. Phys. 35325 (2012)

K. Werner, K. de Vries and O. Scholten, Astropart. Phys. 37 5-16 (2012)

V. Marin and B. Revenu, Astropart. Phys. 35, p. 733-741 (2012).

[2] D. Ardouin et al. (CODALEMA collaboration), Astropart. Phys. 31, 192:200 (2009).

[3] V. Marin et al. (CODALEMA collaboration), 32 ${ }^{\text {nd }}$ ICRC, Beijing, China (2011)

A. Bellétoile et al., Astropart. Phys. 69 50-60 (2015)

The Pierre Auger Collaboration, Phys. Rev. D 114, 165001 (2014).

[4] S. Buitink et al. (LOFAR Coll.), $33^{\text {rd }}$ ICRC, Rio de Janeiro, Brazil (2013).

[5] F. Gaté et al., these proceedings (2015).

[6] J. Schulz, for the Pierre Auger collaboration, these proceedings (2015).

[7] P. Schellart et al. (LOFAR Collaboration), A\&A 560 A98 (2013).

[8] D. Torres-Machado et al. (CODALEMA Coll.), 33 ${ }^{\text {rd }}$ ICRC, Rio de Janeiro, Brazil (2013).

[9] B. Revenu et al., $33^{\text {rd }}$ ICRC, Rio de Janeiro, Brazil (2013).

[10] L. Martin et al., $\sigma^{\text {th }}$ ARENA Conference, Annapolis, USA (2014).

[11] A. Nelles et al., submitted to Astropart. Phys. (2014).

[12] D. Charrier, $6^{\text {th }}$ ARENA Conference, Annapolis, USA (2014).

The Pierre Auger Collaboration, JINST 7, P10011 (2012).

[13] K. de Vries, O. Scholten and K. Werner, Astropart. Phys. 4523 (2013).

[14] P. Zarka, J.N. Girard, M. Tagger et al. (LSS team) (2012), Proc. SF2A-2012, 687-694 